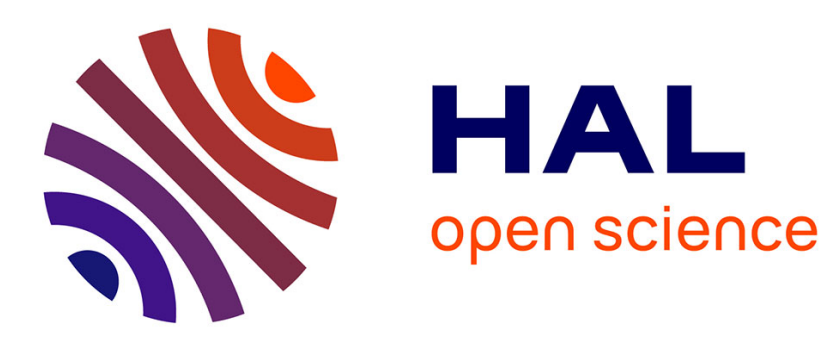

\title{
Utilisabilité d'un Espace Personnel d'Information Modifiable par les Utilisateurs
}

Claudia Detraux, Dominique L. Scapin

\section{To cite this version:}

Claudia Detraux, Dominique L. Scapin. Utilisabilité d'un Espace Personnel d'Information Modifiable par les Utilisateurs. 25ème conférence francophone sur l'Interaction Homme-Machine, IHM'13, AFIHM, Nov 2013, Bordeaux, France. 10.1145/2534903.2534916 . hal-00877290v2

\section{HAL Id: hal-00877290 https://hal.inria.fr/hal-00877290v2}

Submitted on 6 Nov 2013

HAL is a multi-disciplinary open access archive for the deposit and dissemination of scientific research documents, whether they are published or not. The documents may come from teaching and research institutions in France or abroad, or from public or private research centers.
L'archive ouverte pluridisciplinaire HAL, est destinée au dépôt et à la diffusion de documents scientifiques de niveau recherche, publiés ou non, émanant des établissements d'enseignement et de recherche français ou étrangers, des laboratoires publics ou privés. 


\section{Utilisabilité d'un Espace Personnel d'Information Modifiable par les Utilisateurs}

\author{
Claudia Detraux \\ INRIA \\ Domaine de Voluceau \\ 78153, Le Chesnay Cedex \\ claudia.serey@gmail.com
}

\author{
Dominique L. Scapin \\ INRIA \\ Domaine de Voluceau \\ 78153, Le Chesnay Cedex \\ dominique.scapin@inria.fr
}

\begin{abstract}
RESUME
Cet article présente une étude sur l'utilisabilité d'un nouveau prototype de système de gestion des informations personnelles sur Internet (PIMI). Les objectifs sont d'évaluer sa facilité d'utilisation, et d'évaluer la modification utilisateur comme technique d'évaluation. Trente utilisateurs ont participé à l'expérience : une première partie consistait en un test utilisateur classique (TUC) et une seconde partie était un test d'utilisabilité avec modifications utilisateur (TUM). Un total de 51 problèmes d'utilisabilité a été diagnostiqué. Parmi eux, 32 avec TUC, et 19 avec TUM. Une partie de ces derniers (11) s'ajoute à ceux identifiés avec TUC, et à ceux diagnostiqués précédemment lors d'une inspection de l'utilisabilité (IU avec Critères Ergonomiques). La participation active des utilisateurs au travers de scénarios de personnalisation semble fournir des indices supplémentaires pour l'évaluation de l'utilisabilité et pour la conception (nouvelles recommandations génériques d'utilisabilité).
\end{abstract}

\section{Mots Clés}

Utilisabilité ; méthodes d'évaluation ; modifications utilisateur ; e-gov.; informations personnelles.

\section{ACM Classification Keywords}

H.5.m. Information interfaces and presentation ; H.1.2 Human factors ; H.5.2 Evaluation/Methodology.

\section{INTRODUCTION}

Les PIMs (Personal Information Management systems) envahissent notre vie quotidienne, soit comme citoyens, employés, soit dans le cercle familial, amical, etc. Plusieurs types d'outils sont disponibles pour supporter ces activités et ainsi organiser les différentes données personnelles. Ces systèmes se doivent d'être utilisables, notamment pour atteindre l'objectif des initiatives gouvernementales de dématérialisation des procédures.

Dans ce contexte, une étude a été menée au sein du projet ANR PIMI (Personal Information Management through Internet) avec l'objectif d'aider à réaliser

(C) ACM, 2013. This is the author's version of the work. It is posted here by permission of ACM for your personal use. Not for redistribution. The definitive version was published in Actes de la $25^{\text {ième }}$ conférence francophone sur l'Interaction Homme-Machine, 2013.

http://dx.doi.org/10.1145/2534903.2534916 facilement différentes téléprocédures de type egouvernement.

Ce PIMs est plus générique qu'un "profil client", ou que des formulaires spécifiques requis par les différentes procédures. Ce PIMs appartient à l'utilisateur avec l'ensemble de ses données personnelles. Les autres formulaires pourront ainsi être un sous-ensemble de ces données avec des objectifs spécifiques (par exemple : demande de passeport, de bourse étudiant, de logement, paiement des impôts, etc.). Ce PIMs pourra alimenter ces formulaires et "profils clients".

Cette étude fait suite à un travail qui a contribué de manière itérative au prototype actuel. Plusieurs études d'utilisabilité ont été réalisées [27] : une analyse de formulaires administratifs; trois focus groups sur le stockage et le partage d'informations ; un questionnaire en ligne sur les mêmes thèmes pour élargir la population ; un étude de "card-sorting" pour valider les catégories crées par les utilisateurs et aussi leurs façon de les organiser. Une maquette PIMI a ensuite été élaborée. Une autre étude [7] a complémenté cette approche par une expérimentation. Son objectif était de valider le contenu et la structure d'items d'information proposés au travers d'une maquette partiellement modifiable sur requête des utilisateurs. Les résultats ont permis de valider la structure d'items du futur espace personnel, de commencer à analyser les modifications réalisées par l'utilisateur comme indices d'évaluation et de proposer des recommandations pour améliorer son utilisabilité.

L'étude actuelle concerne l'évaluation ergonomique du nouveau prototype PIMI. Une expérimentation a été menée avec 30 participants. Elle se composait d'une partie test utilisateur classique (TUC) et d'une deuxième partie où les utilisateurs ont été invités à modifier le prototype (TUM). L'objectif était double : évaluer l'utilisabilité d'un prototype avancé de PIMI (contenu, structure et leur personnalisation), et étudier le rôle de différentes méthodes dans le diagnostic de problèmes d'utilisabilité. Les résultats du TUC et du TUM ont été comparés. Puis, ces résultats ont été confrontés à une Inspection Ergonomique (IE) réalisée auparavant.

Cet article propose d'abord une revue bibliographique sur les PIMs, les interfaces modifiables, et la comparaison de méthodes d'évaluation de l'utilisabilité. Puis l'expérimentation est décrite (méthode, procédure, 
matériel, participants), ainsi que les principaux résultats obtenus. La conclusion discute des enseignements méthodologiques et de pistes pour le futur.

\section{TRAVAUX CONNEXES}

Personal Information Management systems (PIMs) : le domaine des systèmes de gestion de l'information personnelle se réfère notamment aux études sur la manière dont les utilisateurs gèrent leurs données et documents personnels. Du point de vue de l'utilisabilité, la littérature n'est pas encore très riche. Certains aspects sont couverts en partie dans les conférences de type CSCW [5], bien sûr principalement sur la coopération, les groupes, les interactions sociales, et sur les aspects sociétaux dans [23]. D'autres articles sont plus directement concernés par les utilisateurs, comme «un écosystème numérique personnel de l'utilisateur» [17], ou sur l'évolution de l'utilisation des tablettes [3], ou la vie privée [24] et [25]. Certaines contributions concernent la facilité de remplissage de formulaires dans [32], la recherche sur le web [22], et de permettre la flexibilité entre les périphériques [1]. Certaines études longitudinales ont tenté de comprendre la prise de notes et l'archivage des informations [28], ou les pratiques mobiles [19]. Cependant, peu d'apports nouveaux sur l'évaluation de l'utilisabilité, par rapport à [7, op. cit.] qui, outre un examen de 15 outils PIMs, a évoqué les problèmes d'organisation et de récupération de l'information au sein des structures hiérarchiques, encore les plus répandues et préférées des utilisateurs [9] [13] [14] [29], mais qui montrent aussi les difficultés des utilisateurs avec la création de structures hiérarchiques cohérentes et significatives, ainsi qu'avec la dénomination des catégories et rubriques. L'expérience décrite ici tente d'aller plus loin sur ces questions de structure et de dénomination des informations personnelles.

Modifications Utilisateurs. Un travail précédent [7] a proposé une revue bibliographique sur les interfaces modifiables. Depuis, peu de nouvelles contributions ont été identifiées au delà de [21] [31] [6] [8] qui traitent de la modification utilisateur lors de la conception/ développement de systèmes (notamment échanges entre utilisateurs et concepteurs), son utilisabilité et son impact sur les mesures d'utilisabilité. D'autres articles traitent plutôt de questions comme les systèmes de recommandations [15], réseaux sociaux [32], jeux [10], environnements physiques [19], e-learning [18], etc. En résumé, la modification a plutôt été utilisée jusqu'à maintenant dans la littérature pour aider la communication concepteurs-utilisateurs, mais pas comme moyen d'évaluation de l'utilisabilité. Face à ce manque, cette étude tente d'apporter de nouveaux résultats empiriques sur ce point. Pour cela, le principe a été de proposer à des utilisateurs d'effectuer les modifications qu'ils souhaitaient sur le contenu et la structure du prototype, à leur guise ou selon un scénario.
Méthodes d'Évaluation de l'Utilisabilité : de nombreuses sont proposées dans la littérature (voir [26] et (pour Test Utilisateurs et Inspection) voir ISO 16982 [33], notamment 5.1 Methods which imply the direct involvement of users, et 5.2 Methods which do not imply the direct involvement of users. Leur comparaison est longue et complexe, en particulier lorsque l'on suit des lignes directrices et critères tels que dans [11] [12]. Lors d'un examen approfondi de la littérature [30] diverses questions ont été rapportées (voir [4] [11 et 12 op. cit.] [16]), comme l'utilisation de mesures appropriées, la gravité des problèmes d'utilisabilité, leur authenticité, la rigueur des analyses, les problèmes d'interprétation des problèmes et leur similitude. Dans cette étude, nous avons tenté de pallier ces difficultés grâce à un soin particulier au codage de problèmes. Cependant, alors que nous avons utilisé un assez grand nombre de participants pour l'expérience, l'inspection ergonomique a, quant a elle, été coréalisée par seulement 2 experts, contrairement à l'étude citée [29, op. cit.].

\section{EXPERIMENTATION}

L'objectif de l'expérimentation est d'évaluer le prototype PIMI en obtenant des informations sur le comportement intuitif de l'utilisateur. Les objectifs spécifiques sont de: (1) vérifier l'utilisabilité de l'interface du système pour saisir des informations, retrouver des items, et personnaliser la dénomination des catégories, des rubriques, changer l'ordre d'affichage, masquer des catégories et rubriques; (2) vérifier si la dénomination des catégories, rubriques et items est compréhensible et convient aux utilisateurs; (3) vérifier si la structure proposée est compréhensible et acceptée par les utilisateurs; (4) vérifier si les utilisateurs souhaitent modifier leurs espaces personnels ; et conjointement vérifier si les scénarios de modification apportent des indices additionnels à l'évaluation ergonomique, par rapport aux scénarios classiques.

Les mesures étaient répétées (plan intra); avec les caractéristiques des participants comme variables indépendantes; et le comportement des sujets comme variable dépendante (temps, difficultés, erreurs, etc.). Pas de variation du prototype selon les participants ou les tâches. L'étude étant qualitative, l'analyse a porté essentiellement sur un examen détaillé des opérations effectuées (nature des modifications), les données se prêtant mal à des statistiques inférentielles, à cette étape.

\section{Méthode et procédure}

Les séances ont été réalisées individuellement et accompagnées par l'expérimentateur qui a présenté le projet, expliqué les objectifs de l'expérimentation et le prototype (comment naviguer, saisir, enregistrer et modifier le PIMI) ; questionné les participants; les a incités et motivés à parler de leurs difficultés en rapport aux tâches, à l'outil, aux données. De leur côté, les participants devaient réaliser les tâches demandées par l'expérimentateur, répondre aux questionnaires et 
exprimer leur opinion. Pour mettre les participants en situation, on a choisi de mener les séances dans leur propre environnement de travail (dans leurs bureaux pour les chercheurs et le personnel administratif) ou d'étude (salle d'informatique pour les étudiants). De cette façon, ils pouvaient accéder facilement à leurs effets personnels (sac, portefeuilles, agendas, ordinateurs personnels ou de travail), pour la réalisation des tâches demandées. Le prototype a été présenté sur un ordinateur portable et les séances enregistrées avec un logiciel de capture d'écran (vidéo et son).

Un test pilote a été réalisé avec un sujet de formation ingénieur. Cela a permis de valider la consigne et le discours de l'expérimentateur ainsi que de calibrer la durée des sessions. Les séances se sont déroulées en 3 phases principales:

- Présentation du projet et de l'application (comment naviguer, saisir les données et effectuer les modifications). Les participants sont invités à penser à voix haute pendant l'exécution des tâches.

\section{- Travail sur Scénarios :}

Dans une première partie, 4 scénarios ont été proposés consécutivement: Scénario 1: tâches d'identification personnelle (saisir nom, prénom, informations de la carte d'identité, adresse, téléphone); Scénario 2 : tâches qui concernent les contacts professionnels et le parcours étudiant; Scénario 3: tâches de saisie dans 2 types de formulaires ; Scénario 4 : tâches destinées à vérifier (chemin parcouru) si les redondances proposées sont comprises et utilisées.

Dans une deuxième partie, un des objectifs était d'étudier le caractère modifiable d'un système de ce type par les utilisateurs afin d'adapter leur espace personnel selon leurs besoins et envies. Deux étapes sont proposées: Étape 1 (Modifications par consigne): scénario avec consignes expérimentales de modifications, afin de surtout vérifier la facilité à les réaliser et le(s) chemin(s) utilisés pour les effectuer (fonction «Personnaliser le PIMI» ou «Options de Personnalisation » de «Saisir et Visualiser les Informations »). Étape 2 (Modifications à l'initiative des participants) : scénario demandant de parcourir toute la structure du PIMI et d'effectuer des modifications selon les souhaits des participants.

Finalement dans une troisième partie, les participants ont répondu à un questionnaire composé de 2 sections : la première section comportait des questions spécifiques sur l'aspect modification, pour comprendre l'acceptation de cette étape ainsi que les préférences des utilisateurs pour ce type de modification, ainsi que les difficultés à la réaliser. La deuxième section comportait des questions plus générales sur le système PIMI et sur les améliorations souhaitées. Les questions sont «fermées » (oui/non ou liste d'options). Cependant, l'expérimentateur encourage l'utilisateur à justifier ses réponses oralement (enregistrement audio) ou par écrit.

\section{Participants}

Les séances ont été réalisées avec 30 participants (10 chercheurs, 10 personnels administratifs et 10 étudiants), dont 16 participants masculins $(53,3 \%)$ et 14 participants féminins $(46,6 \%)$. La moyenne d'âge est de 36,06 ans (sd. 16,8). Dans la catégorie «chercheurs », 8/10 des participants sont masculins $(80 \%)$, et $2 / 10$ féminins (20\%). La moyenne d'âge est de 47,5 ans (sd. 17,48). Dans la catégorie «personnel administratif », 9/10 des participants sont féminins $(90 \%)$, et $1 / 10$ masculin. La moyenne d'âge est de 41,2 ans (sd. 11,4). Dans la catégorie «étudiants », 7/10 participants sont masculins $(70 \%)$ et 3 participants féminins $(30 \%)$. La moyenne d'âge est de 19,5 ans (sd. 1,17).

\section{Matériel}

Items d'Information

Les résultats des études précédentes [7] ont abouti à une structure de 73 items d'information, avec 10 catégories, et 35 rubriques (voir Tableau 1).

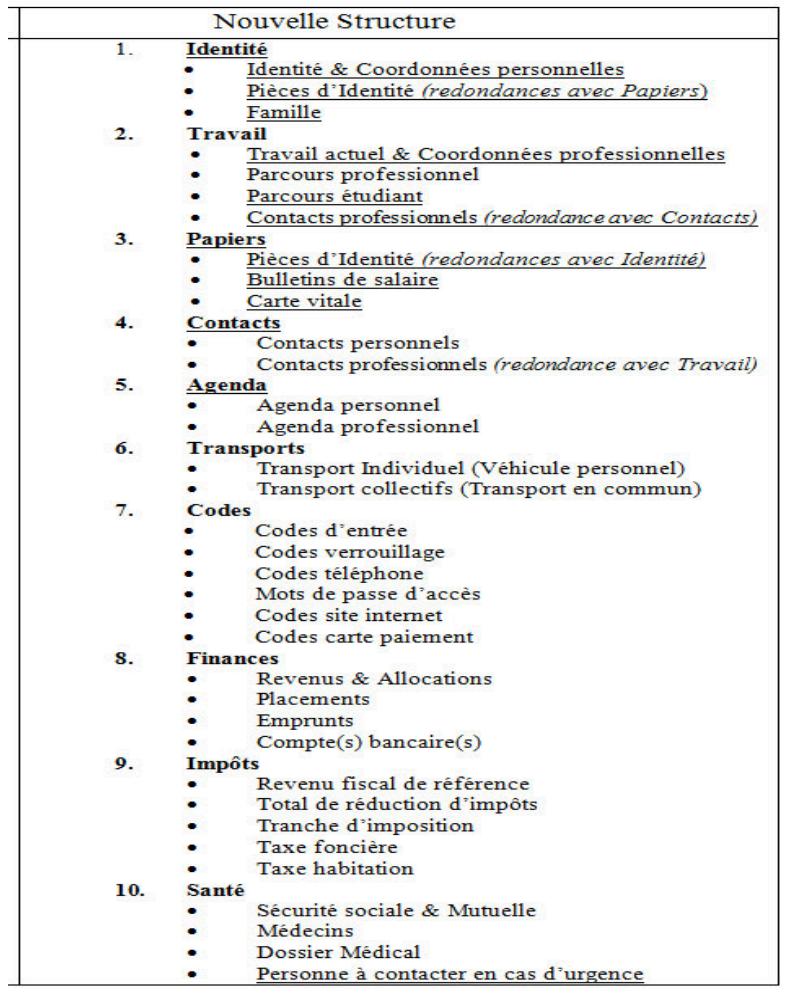

Tableau 1 : Catégories et Rubriques

\section{Matériel Expérimentateur}

Un logiciel de capture video ( $\mathrm{NCH}$ Software) permet d'enregistrer en video (écran) les séances ainsi que les commentaires des participants pendant l'execution des scénarios (utilisé avec la permission des utilisateurs). Des check-lists sont également remplies par 
l'expérimentateur afin de noter les informations les plus pertinentes, et les évènements importants.

\section{Matériel Participants}

Ordinateur portable avec prototype / logiciel de capture installé ; fiches de description des scénarios et tâches à accomplir ; fiches additionnelles pour les cas où l'utilisateur n'a pas sur lui les données à saisir (l'expérimentateur lui fournit une fiche qui simule une carte de visite d'un médecin spécialiste, d'un collègue, etc.) ; fichiers à charger qui sont demandés dans les scénarios, comme : carte d'identité fictive.jpg, parcours universitaire fictif.pdf.; questionnaire final comportant des questions sur l'application, son utilisation et le degré d'adoption d'un tel système. Ce questionnaire comporte une partie spécifique sur la modification et la participation utilisateurs.

\section{Prototype PIMI}

Le prototype évalué (V 0.3 , version $\mathrm{PC}$ ) a été mis à disposition localement et accédé par le navigateur Firefox sur une plateforme Windows. Les Figures 1 et 2 montrent des images capturées du prototype.

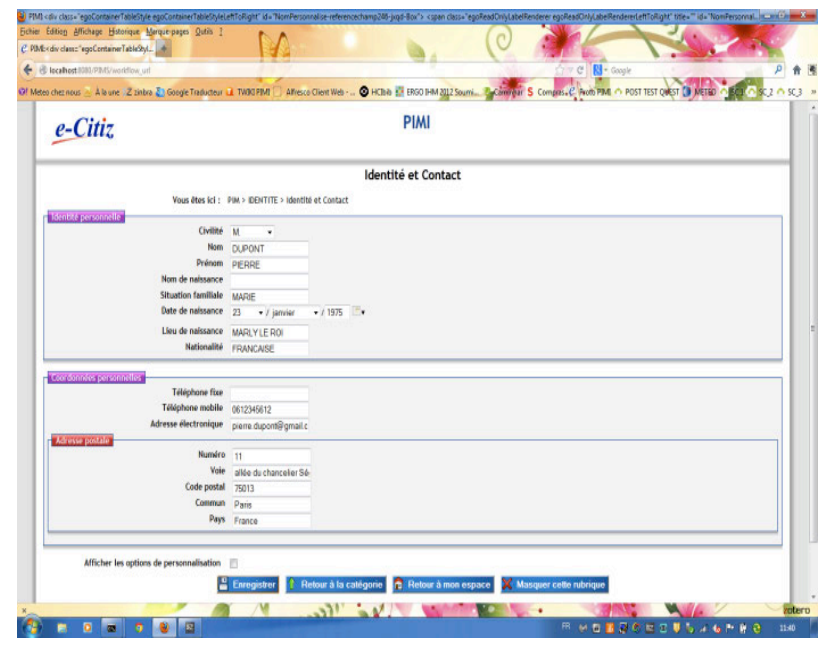

Figure 1. Écran du prototype PIMI (identité et contact)

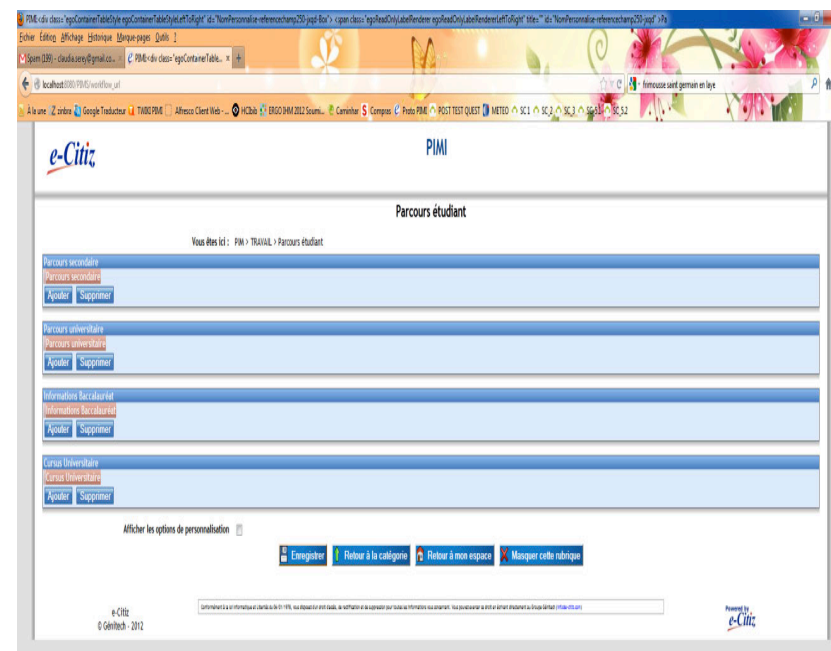

Figure 2. Écran du prototype PIMI (parcours étudiant)

\section{RESULTATS}

Durée des séances : la durée moyenne a été de 46,93 minutes (sd. 10,16). Dans la catégorie « chercheurs », la moyenne est de 46,3 minutes (sd. 8,38) ; pour les «personnels administratifs » de 51,9 minutes (sd. 11,76) ; et pour les « étudiants » de 42,6 minutes (sd. 8,69).

Les analyses sont basées sur les «difficultés» et les « erreurs », ainsi que sur les commentaires des participants. Les difficultés englobent les délais pour trouver une information, une navigation confuse, un temps d'inactivité ; les erreurs sont des actions erronées ou sans lien ; les commentaires sont exprimés par les participants pendant les séances ou lors du questionnaire. La plupart des commentaires renforcent des difficultés ou erreurs observées. On peut remarquer que les problèmes ont été détectés essentiellement à partir des erreurs et difficultés observées (33\%), des commentaires des participants (27\%), et $40 \%$ par les deux moyens.

Groupes de participants (voir Tableau 2) : on constate, pour TUC, des valeurs d'erreurs similaires. Pour TUM, en termes de modifications, les étudiants ont plus modifié (19 dénominations, 37 masquages et 34 ordres, pour un total de 90), tandis que les administratifs en ont effectué moins (16 dénominations, 32 masquages, et 34 ordres, pour un total de 75), et les chercheurs encore moins 50 (7 dénominations, 34 masquages, et 9 ordres, pour un total de 50).

\begin{tabular}{|c|c|c|c|}
\cline { 2 - 4 } \multicolumn{1}{c|}{} & Chercheurs & Administratifs & Étudiants \\
\hline Erreurs TUC & $39 / 124$ & $42 / 124$ & $43 / 124$ \\
\hline Modifs TUM & 50 & 75 & 90 \\
\hline
\end{tabular}

Tableau 2 : Erreurs et modifications par groupe

Globalement, il semble que les utilisateurs les plus jeunes (étudiants) inclinent plus à proposer et adapter leur espace personnel.

Les réponses au questionnaire ont d'ailleurs montré que les seuls avis négatifs sur la facilité d'adaptation (5 participants) viennent de 3 chercheurs et de 2 administratifs.

Difficultés à trouver des informations L'accès aux catégories et sous-catégories n'est pas vraiment un problème, contrairement à trouver certains items individuels (voir Tableau 3).

\begin{tabular}{|c|c|c|}
\hline & Difficultés & Items \\
\hline Scénario 1 & $16,1 \%$ & parents \\
\hline Scénario 2 & $3,2 \%$ & contacts professionnels \\
\hline Scénario 3 & $19,4 \%$ & $\begin{array}{c}\text { sécurité sociale, médecin spécialiste (et } \\
58,1 \% \text { n'ont pas trouvé) }\end{array}$ \\
\hline Scénario 4 & $33,3 \%$ & $\begin{array}{l}\text { «ID» (fichier joint) } \\
\quad 3,3 \% \text { n'ont pas trouvé) }\end{array}$ \\
\hline
\end{tabular}

Tableau 3 : Difficultés à trouver des informations

Problèmes de dénomination. Les termes avec lesquels les participants ont eu quelques difficultés de 
compréhension (voir Tableau 4) semblent correspondre à des termes mal choisis, ou manquant de précision, ou encore présentés sans un contexte suffisant.

\begin{tabular}{|c|c|c|}
\hline Rubrique & Difficultés & Items \\
\hline Identifications & 6 & situation familiale \\
personnelles & 3 & nom de naissance \\
& 1 & lieu de naissance \\
\hline Informations & 11 & numéro \\
collègues & & cursus universitaire et cours \\
\hline Cursus de l'étudiant) & 2 & bénéficiaire \\
\hline Sécurité sociale & 8 & période de couverture \\
& 25 & nombre \\
\hline Autres spécialistes de & 28 & nom personnalisé \\
santé & & \\
\hline
\end{tabular}

Tableau 4 : Difficultés liées à la dénomination

Difficultés de navigation (voir Tableau 5) : 19/30 participants ont eu au moins une difficulté, un seul en a eu 4 (total 33, moyenne 1,1 ; sd. 1,15). Les problèmes d'utilisabilité sont principalement liés à certains boutons de navigation et au manque de raccourcis.

\begin{tabular}{|c|c|c|}
\cline { 2 - 3 } \multicolumn{1}{c|}{} & Difficultés & Erreurs \\
\hline Scénario 1 & 7 & 3 \\
\hline Scénario 2 & 5 & 2 \\
\hline Scénario 3 & 4 & 2 \\
\hline Scénario 4 & 5 & 1 \\
\hline Modifs 1 & 7 & 3 \\
\hline Modifs 2 & 5 & 4 \\
\hline
\end{tabular}

Tableau 5 : Difficultés de navigation

Enregistrement de formulaires (voir Tableau 6). Cette opération donne lieu à 30 difficultés : $70 \%$ des participants ont eu au moins 1 difficulté, et 3 participants ont eu 3 difficultés. Les problèmes d'utilisabilité sont principalement liés au manque de feedback, et à des confusions de dénomination.

\begin{tabular}{|c|c|c|}
\cline { 2 - 3 } \multicolumn{1}{c|}{} & Difficultés & Erreurs \\
\hline Scénario 1 & 13 & 2 \\
\hline Scénario 2 & 7 & 4 \\
\hline Scénario 3 & 9 & 2 \\
\hline Scénario 4 & \multicolumn{2}{c|}{ Non concerné } \\
\hline Modifs 1 & 0 & 0 \\
\hline Modifs 2 & 0 & 1 \\
\hline
\end{tabular}

Tableau 6 : Difficultés d'enregistrement de formulaires

Globalement, les erreurs confirment les constatations précédentes. Un total de 124 erreurs a été observé lors de l'expérience (moyenne 4,13 par participant, sd. 2,17) : Scénario 1 (moyenne 1,13, sd. 1,25), avec au moins 1 erreur pour 18 participants, et 5 pour 1 participant ; Scénario 2 (moyenne 1,3, sd. 1,2), avec au moins 1 erreur pour 20 participants et 4 pour 1 participant ; Scénario 3 (moyenne 1,3, sd. 1,05), avec au moins 1 erreur pour 23 participants et 5 pour 1 participant ; Scénario 4 : seulement 1 erreur ; Scénario 5.1: 3 erreurs pour 3 participants (1 chacun) ; Scénario $5.2: 8$ erreurs (moyenne 0,26 ; sd. 0,69 ), avec au moins 1 erreur pour 5 participants, et 3 pour 1 .

\section{Personnalisation : Modifications par consigne}

Chemins parcourus : pour le changement de dénomination: 63,3 \% des participants (19/30) sont partis directement à la fonction « Personnaliser le PIMI » tandis que $6,7 \%$ des participants (2/30) ont choisi d'aller dans chaque rubrique. Les autres $30 \%$ des participants (9/30) ont utilisé les deux chemins (d'abord par les rubriques et ensuite par la fonction «Personnaliser le PIMI »).

\section{Difficultés :}

- Pour le changement des dénominations : $90 \%$ (27/30 participants) ont effectué facilement les modifications, 2 participants $(6,7 \%)$ ont pris un peu de temps pour les réaliser, et seulement $1(3,3 \%)$ n'a pas réussi.

- Pour le masquage : 56,7 \% des participants (17/30) ont effectué facilement le masquage des catégories et rubriques, 11 participants $(36,7 \%)$ ont pris un peu de temps pour le réaliser, et $2(6,7 \%)$ n'ont pas réussi.

- Pour le changement d'ordre : 43,3\% des participants $(13 / 30)$ ont effectué facilement le masquage des catégories et rubriques, et 17 participants $(36,7 \%)$ ont pris un peu de temps pour le réaliser.

- Pour la restauration de la structure par défaut : 96,7\% des participants (29/30) ont effectué facilement la restauration par défaut de la structure, 1 seul participant $(3,3 \%)$ ayant pris un peu de temps pour la réaliser.

\section{Personnalisation: Modifications à l'initiative des participants}

Le Tableau 7 montre un total de 222 modifications suggérées (Chercheurs $\mathrm{C} 1$ à $\mathrm{C} 10$; Administratifs $\mathrm{A} 1$ à A10 ; Étudiants, E1 à E10), avec une moyenne de 7,4 par participant (sd. 4,79).

Tous les participants ont effectué des modifications. La personne qui a fait le plus de modifications en a suggéré 19 alors que 4 personnes en ont suggéré seulement 1 . Le Tableau 7 montre que le type de modification le plus fréquent est le masquage (catégories, rubriques et items) (103 modifications), suivi du changement d'ordre des catégories (77 modifications), puis le changement des dénominations (42 modifications).

- Pour le masquage, 58 modifications ont concerné les catégories (93,3\% participants), 22 les rubriques (14,3\% participants), et 23 les items (3,2\% participants).

- Pour les modifications d'ordre, 50\% des participants ont effectué au moins 2 modifications (maximum 9). 


\begin{tabular}{|c|c|c|c|c|c|c|c|c|}
\hline & $\begin{array}{c}\text { Dénomination } \\
\text { Categories }\end{array}$ & $\begin{array}{c}\text { Denomination } \\
\text { Rubriques }\end{array}$ & $\begin{array}{c}\text { Masquage } \\
\text { Catégories }\end{array}$ & $\begin{array}{c}\text { Masquage } \\
\text { Rubriques }\end{array}$ & $\begin{array}{c}\text { Masquage } \\
\text { Items }\end{array}$ & $\begin{array}{c}\text { Ordre } \\
\text { Catégories }\end{array}$ & Total \\
\hline C1 & 0 & 3 & 1 & 1 & 1 & 0 & 6 \\
\hline C2 & 1 & 0 & 1 & 0 & 0 & 0 & 2 \\
\hline C3 & 0 & 0 & 2 & 3 & 0 & 0 & 5 \\
\hline C4 & 0 & 2 & 1 & 2 & 0 & 4 & 9 & \\
\hline C5 & 0 & 0 & 5 & 2 & 0 & 3 & 10 \\
\hline C6 & 0 & 1 & 0 & 0 & 0 & 0 & 1 \\
\hline C7 & 0 & 0 & 2 & 0 & 5 & 0 & 7 \\
\hline C8 & 0 & 0 & 1 & 0 & 0 & 0 & 1 \\
\hline C9 & 0 & 0 & 3 & 1 & 0 & 2 & 6 \\
\hline C10 & 0 & 0 & 2 & 1 & 0 & 0 & 3 \\
\hline A1 & 0 & 0 & 0 & 0 & 0 & 5 & 5 \\
\hline A2 & 4 & 1 & 4 & 2 & 0 & 0 & 11 \\
\hline A3 & 1 & 2 & 3 & 2 & 0 & 9 & 17 \\
\hline A4 & 0 & 0 & 1 & 0 & 0 & 4 & 5 \\
\hline A5 & 2 & 0 & 3 & 0 & 0 & 8 & 13 \\
\hline A6 & 2 & 1 & 1 & 0 & 0 & 4 & 8 \\
\hline A7 & 0 & 0 & 4 & 0 & 0 & 0 & 4 \\
\hline A8 & 2 & 1 & 1 & 0 & 0 & 4 & 8 \\
\hline A9 & 0 & 0 & 1 & 0 & 0 & 0 & 1 \\
\hline A10 & 0 & 0 & 4 & 0 & 6 & 0 & 10 \\
\hline E1 & 1 & 0 & 2 & 0 & 5 & 0 & 8 \\
\hline E2 & 3 & 2 & 1 & 1 & 0 & 0 & 7 \\
\hline E3 & 2 & 0 & 1 & 1 & 0 & 2 & 6 \\
\hline E4 & 0 & 0 & 1 & 0 & 0 & 0 & 1 \\
\hline E5 & 0 & 0 & 1 & 0 & 0 & 8 & 9 \\
\hline E6 & 1 & 1 & 2 & 2 & 6 & 4 & 16 \\
\hline E7 & 0 & 0 & 1 & 0 & 0 & 4 & 5 \\
\hline E8 & 0 & 1 & 3 & 1 & 0 & 0 & 5 \\
\hline E9 & 2 & 3 & 5 & 2 & 0 & 7 & 19 \\
\hline E10 & 1 & 2 & 1 & 1 & 0 & 9 & 14 \\
\hline TOTAL & 22 & 20 & 58 & 22 & 23 & 77 & \\
\hline MOYENNE & 0,73 & 0,66 & 1,93 & 0,73 & 0,76 & 2,5 & \\
\hline ECART-TYPE & 1,08 & 0,95 & 1,38 & 0,91 & 1,9 & 3,1 & \\
\hline & & & & & & & \\
\hline
\end{tabular}

Tableau 7 : Modifications par groupes de participants

Enfin, $47 \%$ des participants ont exprimé le besoin d'un ensemble de modifications non proposées : ajout de catégories ou sous-catégories (33\%) ; division d'une catégorie $(0,6 \%)$, déplacement de sous-catégories $(0,6 \%)$ ; distinction entre entrée de données et récupération de données $(0,3 \%)$, modification des codes couleur, de la taille des caractères $(0,6 \%)$.

\section{Questionnaire}

Section 1 : questions sur la personnalisation

La plupart des participants (83.3\%) ont apprécié de pouvoir modifier leur Espace Personnel d'information. La plupart $(93,3 \%)$ ont également trouvé qu'il était facile de modifier les noms des catégories et des souscatégories $(80 \%)$. Il leur a également semblé facile de masquer des catégories et sous-catégories $(93,3 \%)$. Il a été jugé beaucoup moins facile de modifier l'ordre des catégories $(36,7 \%)$. En outre, les participants ont suggéré d'autres types de modifications qui n'ont pas été mises en œuvre dans le prototype, telles que : ajouter de nouvelles catégories et sous-catégories $(9 / 20)$; changer le design visuel, par exemple, les couleurs, les formats (4/30); et fournir les formulaires horizontalement (2/30).

\section{Section 2 : questions générales et améliorations}

Une courte majorité des participants $(60 \%)$ se sent prêt à utiliser un tel PIMI. Leur motivation est essentiellement la capacité à centraliser toutes leurs données et de limiter la consommation de papier. Pour ceux qui se sentent moins prêts $(33,3 \%)$ et ceux qui n'étaient pas prêts du tout $(6,7 \%)$, la principale préoccupation est la sécurité et la confiance. La plupart des participants $(80 \%)$ ont dit ne pas avoir éprouvé de difficultés pour utiliser le prototype. Seules ont été exprimées quelques difficultés modérées $(16,7 \%)$ ou fortes $(3,3 \%)$. En outre, 25/30 participants $(86,2 \%)$ ont fait des suggestions d'amélioration, essentiellement pour atténuer certains des problèmes d'utilisabilité mentionnés précédemment.

D'un point de vue méthodologique, il est à remarquer que, globalement, les diverses évaluations d'utilisabilité menées dans cette étude ont conduit au diagnostic d'un plus grand ensemble de problèmes d'utilisabilité que les seules réponses au questionnaire. Toutefois, celles-ci, en particulier les suggestions ont confirmé une partie des problèmes identifiés.

\section{DISCUSSION}

Personnalisation : La participation lors de cette session a été forte : un total de 222 modifications ont été apportées, et tous les participants ont fait au moins un changement avec un maximum de 19 pour un participant (moyenne 7,4, sd. 4,79).

Les modifications peuvent être caractérisées selon 4 buts principaux : simplifier (par masquage et changement de dénomination), clarifier (par changement de nom), faciliter l'accès (par changement d'ordre des catégories); sécuriser (par masquage).

Quelques enseignements méthodologiques peuvent être tirés des modifications des participants :

- Cela permet d'identifier des problèmes d'utilisabilité supplémentaires.

- Les participants ont tendance à considérer leur rôle comme plus important, à s'exprimer plus, à fournir des critiques, des suggestions et des préférences. En bref, ils s'approprient le système, voire, renforcent leur opinion sur certains problèmes d'utilisabilité qui ne semblent pas trop "gênants" initialement.

- Compte tenu de la nécessité pour les systèmes actuels d'e-gouvernement d'être utilisés par un public large et varié en termes d'âge, de sexe, de profession, de compétences et de contextes, il est utile de fournir des fonctions de personnalisation par les utilisateurs (par exemple, pour le vocabulaire, les techniques, les besoins de navigation).

En résumé, faciliter les utilisateurs dans un rôle d'acteurs plutôt que d'utilisateurs passifs semble prometteur.

Comparaison de méthodes d'évaluation de l'utilisabilité : la Figure 3 montre le nombre de problèmes d'utilisabilité diagnostiqués avec les 3 méthodes d'utilisabilité (test d'utilisabilité classique (TUC), test d'utilisabilité avec modifications (TUM) et inspection de l'utilisabilité (IU). 


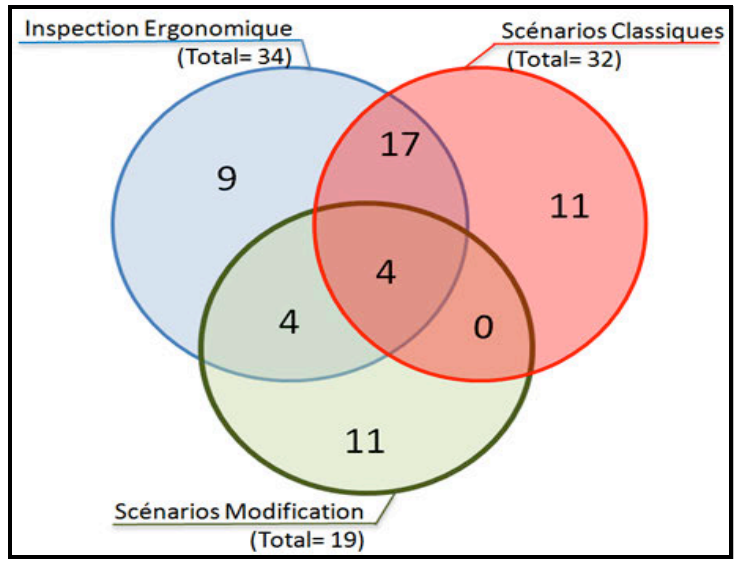

Figure 3 - Problèmes d'utilisabilité par méthode

Globalement, il n'y a pas de différences marquantes dans la nature des problèmes d'utilisabilité, si ce n'est, évidemment, celles liées aux différents états d'interaction parcourus, du fait des scénarios de tâches (états de modification pour TUM et états de saisies de données pour TUC). Toutefois, on trouve :

- Environ le même nombre de problèmes d'utilisabilité spécifiques pour chaque technique d'évaluation : 9 pour IU (26\% du total) ; 11 pour TUC (34\% du total), 11 pour TUM (58\% du total). Cependant, en pourcentage, TUM a le score relatif le plus élevé de problèmes spécifiques.

- Beaucoup (meilleur score) de problèmes communs à IU et TUC ( 21 , soit $62 \%$ de IU et $66 \%$ de TUC).

- Bien moins de problèmes communs à IU et TUM (8, soit $24 \%$ de UI et $42 \%$ de TUM).

- Encore moins de problèmes communs à TUC et TUM (4, c'est-à-dire $12 \%$ de TUC et $21 \%$ de TUM).

- Très peu de problèmes communs aux 3 méthodes IU, TUC et TUM (4, c'est-à-dire $12 \%$ de IU, $12 \%$ de TUC et $21 \%$ de TUM).

En outre, on peut noter que:

- Les 4 problèmes communs à TUC et TUM ont aussi été diagnostiqués avec IU.

- 17 problèmes (sur 21 problèmes communs) ont été diagnostiqués avec TUC et IU, mais seulement 4 ont également été diagnostiqués avec TUM.

- 4 problèmes (sur 8 problèmes communs) ont été diagnostiqués à la fois avec TUM et IU, et 4 avec TUC.

Contrairement aux conclusions de [2], TUC et TUM ne semblent pas conduire à une plus grande diversité de problèmes d'utilisabilité. Cependant, on observe que TUC et TUM semblent particulièrement efficaces pour le diagnostic des problèmes qui nécessitent un état particulier d'interaction pour être détectables. D'un autre côté, IU semble aider à l'identification de problèmes basiques, directement observables, souvent liés à la facilité d'apprentissage et d'utilisation.

\section{CONCLUSION}

Cet article a présenté un travail de recherche associé à l'évaluation de l'utilisabilité d'un prototype pour la gestion des données personnelles. L'expérience, avec 30 participants (chercheurs, administratifs et étudiants), a suivi 4 scénarios de tâches classiques de tests utilisateurs et 1 scénario permettant l'adaptation de la structure de l'information de prototype et son contenu par les participants.

Certaines limites doivent être mentionnées, notamment :

- Pour TUC et TUM, la présence de l'expérimentateur a pu entraver l'accès à certaines fonctions du prototype (par exemple, aide en ligne) car il était juste plus simple de demander à l'expérimentateur.

- Sauf dans le scénario de modification, les participants sont restés concentrés sur les tâches, sans explorer davantage. Un suivi des utilisateurs sur des périodes plus longues pourrait être utile.

- Un ensemble varié de problèmes d'utilisabilité a été identifié. Cependant, il n'y avait aucun contrôle sur les défauts d'utilisabilité résultant (involontairement) de la conception par une entreprise partenaire spécialisée dans les applications e-gouvernement et les outils de conception. Cela empêche bien sûr de généraliser pleinement à toutes les autres applications de ce type. Dans l'avenir, il serait intéressant d'introduire volontairement un grand nombre de défauts d'utilisabilité sur tous les états de l'application, avec une variation complète des types de problèmes, afin d'avoir un contrôle sur le nombre total de problèmes, leur nature et leur emplacement, ce qui permettrait d'extraire l'ensemble des diagnostics en fonction de chaque technique d'évaluation ... mais cela est assez complexe et coûteux, notamment en temps.

- Seuls la structure de l'information et le contenu du prototype ont été évalués, pas les procédures électroniques associées, qui ne seront mises en œuvre que plus tard cette année.

Les résultats ont permis le diagnostic de 51 problèmes d'utilisabilité et ont abouti à des recommandations pour améliorer la version finale du prototype.

D'un point de vue méthodologique, nous avons étudié le rôle relatif des deux méthodes d'évaluation utilisées dans l'expérience (TUC et TUM). En outre, les résultats ont également été comparés aux résultats d'une inspection de l'utilisabilité (IU).

Les trois techniques d'évaluation sont tout à fait complémentaires, mais la participation directe des utilisateurs par personnalisation est une méthode additionnelle utile, permettant d'identifier des problèmes d'utilisabilité spécifiques, notamment de nouveaux chemins, diverses utilisations de fonctions, et différentes compréhensions des concepts.

Bien que l'inspection basée sur les critères ergonomiques ait permis de faire un diagnostic général des problèmes 
d'utilisabilité (plusieurs types et dans de nombreux états de l'interface), le test utilisateur a permis d'identifier des problèmes similaires, mais avec plus de détails, bien que sur un plus petit nombre d'états d'interaction.

L'utilisation d'un scénario de personnalisation permet aux utilisateurs de s'exprimer davantage sur le système en indiquant leurs préférences et ainsi de promouvoir une évaluation et une conception plus collaborative.

Dans l'ensemble, la participation active et directe des utilisateurs, en offrant de nouvelles structures, modalités, chemins et fonctions, sont des indices additionnels très riches pour l'évaluation et la conception.

Pour des travaux de recherche futurs, un certain nombre de questions semblent intéressantes à étudier :

- La sécurité et la confiance est toujours un problème, même si une perception positive semble facilitée par l'implication des utilisateurs dans l'adaptation de la structure et du contenu. Il conviendrait de valider cela pour des e-procédures complètes.

- L'usage réel : il faudra trouver des moyens efficaces de l'évaluer grâce à des outils innovants et intelligents pour la collecte des données utilisateur en temps réel, y compris sur l'évolution des personnalisations au cours du temps.

- La mobilité : le futur système PIMI devrait être également utilisé sur des smartphones et autres tablettes. Une version PIMI de mobilité est en cours d'élaboration, mais sa facilité d'utilisation et la compatibilité avec la version PC, y compris sa plasticité devront être évaluées.

\section{REMERCIEMENTS}

Cette étude a été menée par INRIA, contrat ANR-PIMI.

\section{BIBLIOGRAPHIE}

1. Ardito, C., Costabile, M. F., Desolda, G., Matera, M., Piccinno, A., and Picozzi, M. Composition of situational interactive spaces by end users: a case for cultural heritage Living history Proc. 7th Nordic Conference on HCI 2012-10-14 p.79-88.

2. Bach, C. and Scapin, D. L.. Comparing Inspections and User Testing for the Evaluation of Virtual Environments, in "International Journal of HumanComputer Interaction", 2010, vol. 26, no 8, p. 786824

3. Buttfield-Addison, P., Lueg, C., Ellis, C., and Manning, J, "Everything goes into or out of the iPad": the iPad, information scraps and personal information management. Proc. of 2012 Australian CHI Conference 2012-11-26 p.61-67.

4. Cockton, G., Woolrych, A., Hall, L., and Hidemarch, M., 2003, Changing Analysts' Tunes: The Surprising Impact of a New Instrument for Usability Inspection Method Assessment. In Proceedings of People and
Computers XVII : Designing for Society, Springer Verlag, pp. 145-162.

5. CSCW13-1. Proceedings of the 2013 ACM Conference on Computer Supported Cooperative Work v.1 / Amy Bruckman / Scott Counts / Cliff Lampe / Loren Terveen San Antonio, Texas 2013-0223 2013-02-27 2013 ACM n.141 p.1566.

6. Demunieux, R., Ganneau, V., Calvary, G., and Gegovska, E. Les interfaces plastiques : premiers retours utilisateurs, évaluations en laboratoire .ErgoIA 2008. 117-124.

7. Detraux, C. and Scapin, D. L. La modification utilisateur comme indice ergonomique évaluatif d'un Espace Personnel d'Information, in "Ergo'IHM 2012, Biarritz, France, 17-19 Octobre 2012.

8. Dörner, C., Yetim, F., Pipek, V., and Wulf, V. "Supporting business process experts in tailoring business processes". Interact. Comput. 23, 3 (2011), 226-238.

9. Evequoz, F. and Lalanne, D. "I thought you would show me how to do it - studying and supporting PIM strategy changes". PIM Workshop, ASIS\&T 2009, $35-42$.

10. Gamberini, L., Spagnolli, A., Carradi, N., Jacucci, G., Tusa, G., Mikkola, T., Zamboni, L., and Hoggan, E. Tailoring Feedback to Users' Actions in a Persuasive Game for Household Electricity Conservation. Proceedings of the 2012 International Conference on Persuasive Technology 2012-06-06 p.100-111

11. Gray, W. D. and Salzman, M. C., 1998, Damaged merchandise? A review of experiments that compare usability evaluation methods. Human-Computer Interaction, 13, pp. 203-261.

12. Hartson, H. R., Andre, T. S. and Williges, R. C., 2001, Criteria for evaluating usability evaluation methods. International Journal of Human-Computer Interaction, 13, pp. 373-410.

13. Henderson, S. "Guidelines for the design of personal document management user interfaces". PIM Workshop, ASIS\&T 2009, 2009, pp. 65-72.

14. Henderson, S. and Srinivasan, A. "An empirical analysis of personal digital document structures", Proc. HCI International 2009, Berlin, Heidelberg: Springer-Verlag, 2009, pp. 394-403.

15. Heung-Nam, K., Majdi, R., and El Saddik, A. Tailoring recommendations to groups of users: a graph walk-based approach. Proceedings of the 2013 International Conference on Intelligent User Interfaces 2013-03-19 v.1 p.15-24

16. Hornbæk, K. and Frøkjær, E., 2005, Comparing usability problems and redesign proposals as input to practical systems development. In Proceedings of 
ACM Conference on Human Factors in Computing Systems, (New York, NY : ACM Press), pp. 391-400.

17. Kay, J., Kummerfeld, B. Creating personalized systems that people can scrutinize and control: Drivers, principles and experience. ACM Transactions on Interactive Intelligent Systems 201212 v. 2 n.4 p.24.

18. Kolås, L. \& Staupe, A. (2007). A Personalized Elearning Interface. In : The Proceedings of the international IEEE Conference on Computer as a tool. Eurocon 2007 (pp. 2670-2675). Poland : IEEE Computer Society.

19. Lindroth, T., and Bergquist, M. Breadcrumbs of interaction : situating personal information management Proc. Fifth Nordic Conference on HCI 2008-10-10 p.266-273.

20. Mirri, S., Salomoni, P., and Prandi, C. Augment browsing and standard profiling for enhancing web accessibility Proceedings of the 2011 International Cross-Disciplinary Conference on Web Accessibility (W4A) 2011-03-28 v.2 p.5.

21. Mørch, A. I., and Mehandjiev, N. D. "Tailoring as collaboration : the mediating tole of multiple representations and application units". Computer Supported Cooperative Work. 9(1) (2000) : 75-100.

22.Nitsche, M., and Nuernberger, A. InFrame-Browsing : Enhancing Standard Web Search Poster Papers. Proc. of Symposium on HCI and Information Retrieval 2012-10-04 p.16.

23. Oakley, R. L. and Salam, A. F. Cyber Citizens and Cyber Deviance : Exploring Social and Technical Factors as Antecedents to Cyber Deviance and the Implications for Cyber Citizenship. Proceedings of the 2012 AIS SIGHCI Workshop on HCI Research in MIS 2012-12-16 p.7.

24. Ploderer, B. Smith, W., Howard, S., Pearce, J., and Borland, R. Things you don't want to know about yourself: ambivalence about tracking and sharing personal information for behaviour change. Proc. of 2012 Australian Computer-Human Interaction Conference 2012-11-26 p.489-492.

25. Rawassizadeh, R. Towards sharing life-log information with society / Behaviour and Information Technology 2012-11 v.31 n.11 p.1057-1067.
26. Scapin, D. L. Law, E., Bevan, N. and the WG1 contributors (2008). Final Report MAUSE COST 294 WG 1 : Critical Review and Analysis of Individual UEMs. COST-294 Project MAUSE (December 2008).

27.Scapin, D. L., Marie-Dessoude, P., Winckler, M., and Detraux, C. Personal Information Systems : User Views and Information Categorization, in "Proc. Centric2011-4th. Intl. Conf. on Advances in Humanoriented and Personalized Mechanisms, Technologies, and Services", Barcelona, Spain, 2011.

28. Van Kleek, Max G., Styke, W., Schraefel, M. C., Karger, D. Finders/keepers : a longitudinal study of people managing information scraps in a micro-note tool Reading \& writing / Proc. ACM CHI 2011 201105-07 v.1 p.2907-2916.

29. Voit, K., Andrews, K. and Slany, W. "Why personal information management (PIM) technologies are not widespread”. PIM Workshop, ASIS\&T 2009. (2009) 60-64.

30. Winckler, M., Gaits, V., Vo, D.-B., Firmenich, S., and Rossi, G. An approach and tool support for assisting users to fill-in web forms with personal information New frontiers in documentation II / ACM 29th Intl. Conf. on Design of Communication 2011-10-03 p.195-202.

31. Zhu, L., Vaghi, I., Barriceli, B. R. “A meta-reflective wiki for collaborative design". Proc. of the 7th International Symposium on Wikis and Open Collaboration (WikiSym'11).ACM, (2011), 53-62.

32. Zimmerman, A., And Lorentz, A. LISTEN : a useradaptive audio-augmented museum guide Special issue on Personalizing Cultural Heritage Exploration. User Modeling and User-Adapted Interaction 200811 v. 18 n.5 p.389-416

33. ISO/TR 16982 (2002). Ergonomics of human-system interaction - Usability methods supporting humancenred design. International Standards Organization, 2002 